\title{
Asymptotic Normality of the Deconvolution Kernel Density Estimator based on Independent Right Censored Data
}

\author{
Wenqing Zhu ${ }^{1} \cdot$ Shan Sun $^{1}$ (D) $\cdot$ Pei Wang $^{1} \cdot$ Hrishabh Khakurel $^{1}$
}

Received: 30 April 2021 / Revised: 27 December 2021 / Accepted: 11 January 2022 /

Published online: 24 February 2022

(c) The Author(s), under exclusive licence to Springer Science+Business Media LLC, part of Springer Nature 2022

\begin{abstract}
We consider estimation of a density when observed lifetime from the convolution model is contaminated by additive measurement errors. A kernel type deconvolving density estimator of the unknown distribution is proposed using Inverse-Probabilityof-Censoring Weighted Average. Further, we discuss the asymptotic normality of the deconvolution kernel density estimator when the error distribution is either ordinary smooth or supersmooth.
\end{abstract}

Keywords Asymptotic normality · Deconvolution · Kernel density · Censored data

\section{Introduction}

Consider a model where variable $Z$ is measured with an additive error $E$, denoted it by

$$
X=Z+E
$$

Model (1) is a well-known deconvolution model where $Z$ is contaminated in $X$ (Fan [4]). An application of this deconvolution model was provided by Comte et al. [2], where the true spontaneous age of menopause $Z$ is always unknown, but it can be

\footnotetext{
$凶 \quad$ Shan Sun

shan.sun@uta.edu

Wenqing Zhu

wkkw_240686@163.com

Pei Wang

pei.wang@mavs.uta.edu

Hrishabh Khakurel

hrishabh.khakurel@mavs.uta.edu

1 Department of Mathematics, The University of Texas at Arlington, Arlington, TX 76019, USA
} 
estimated with an error to obtain the measurement $X$, that is the age at menopause. Furthermore, age at menopause may be censored because of medical intervention. To make inferences about the parameters of $f_{Z}$, such as mean or variance of $Z$, one needs to have an estimator for $f_{Z}$. Another example of model (1) is by Chakrabarty [1] in the study of duration breastfeeding data.

In general, we consider the case of right censored situation: $\{\min (X, C), \mathrm{I}(X \leq C)\}$, where the variable $X$ denotes the true time between the origin and the occurrence of the event of interest and the variable $C$ denotes the true time between the origin and the occurrence of censoring. We assume "random censoring", that is, $X$ and $C$ are independent.

Let us denote the observable random samples in model (1) as

$$
W_{j}=\min \left(Z_{j}+E_{j}, C_{j}\right)=\min \left(X_{j}, C_{j}\right), \text { and } \delta_{j}=\mathrm{I}\left(X_{j} \leq C_{j}\right), j=1,2, \ldots, n
$$

We assume censoring indicator $\delta$ follows a Bernoulli distribution: $P(\delta=1)=p$ and $P(\delta=0)=1-p$, where $0<p<1$.

The first objective of the present study is to provide a non-parametric estimator of the unknown $p d f f_{Z}$ of the unobservable random variable $Z$. The estimator of the $f_{Z}$ can be constructed by using the information based on the observable random sample $\left\{W_{j}, \delta_{j}\right\}$. Detailed formulation of the estimator and the literature related to the properties of the estimator are provided in Sect. 2. Note that the estimation problem in this paper is in the setting of deconvolution which has been widely studied in various contexts. Assuming the density of the error E is known, non-parametric kernel estimators have been proposed by Stefanski and Carrol [14], and Fan [4] when the observations are uncensored. We extend the estimators proposed by Stefanski and Carrol [14] and Fan [4] for censored observations. Furthermore, Fan [4] pointed out that even though the error distribution is assumed to be known, the rate of convergence of the nonparametric deconvolving density estimator of $f_{z}$ depends heavily on the smoothness of the error distribution. Hence, Fan [4] classified the error distribution into two classes: either a class of ordinary smooth distributions or a class of super smooth distributions. Examples of ordinary smooth distributions include $N(0,1)$ and Cauchy $(0,1)$, while $\frac{\alpha^{p}}{\Gamma(p)} x^{p-1} \mathrm{e}^{-\alpha x}$ (Gamma) and $\frac{1}{2} \mathrm{e}^{-|x|}$ (Double exponential) are some of the examples of super smooth distributions. Definitions of these two classes of error distributions are given in Assumption (3.1) in Sect. 3 where $\beta$ controls the rate of convergence of the estimator.

The second and main objective of this paper is to investigate the limiting distribution of the estimator. The main theorems and their proofs are included in Sect. 3. Simulation results of the proposed estimator and their mean integrated squared errors are provided in Sect. 4 for finite sample sizes. 


\section{Non-parametric Estimator}

Recall that in model (1), random sample $\left\{Z_{i}, i=1,2, \ldots, n\right\}$ is contaminated (unobservable) in $X_{i}=Z_{i}+E_{i}$, where $\left\{W_{i}=\min \left(X_{i}, C_{i}\right), i=1,2, \ldots, n\right\}$, and $\left\{E_{i}, i=1,2, \ldots, n\right\}$ are error terms that are independent of $\left\{\delta_{i}=\mathrm{I}\left(X_{i} \leq C_{i}\right), i=1,2, \ldots, n\right\}$. Furthermore, we assume that $E_{i}$ 's has a known pdf $f_{E}$.

To estimate $f_{Z}$, note that we have the following convolution $f_{X}=f_{Z} * f_{E}$, and

$$
\varphi_{f_{Z}}(t)=\frac{\varphi_{f_{X}}(t)}{\varphi_{f_{E}}(t)}
$$

where $f_{X}$ is the $p d f$ of $X$ and $\varphi_{g}$ is the characteristic function of $g$. By Fourier inversion theorem, the estimator of $f_{Z}$ can be written as

$$
\hat{f}_{Z}(z)=\frac{1}{2 \pi} \int \mathrm{e}^{-\mathrm{i} t z} \frac{\varphi_{\hat{f}_{X}}(t)}{\varphi_{f_{E}}(t)} \mathrm{d} t
$$

where $\varphi_{\hat{f}_{X}}(t)=\int \mathrm{e}^{(\mathrm{i} t x)} \hat{f}_{X}(x) \mathrm{d} x$, and $\hat{f}_{X}$ is an estimator for unknown $p d f f_{X}$.

One way to obtain $\hat{f}_{X}$ is via Kaplan-Meier $(\mathrm{K}-\mathrm{M})$ estimator $\hat{F}_{\mathrm{KM}}$

$$
\begin{aligned}
\hat{f}_{X}^{\mathrm{KM}}(x) & =\frac{1}{h} \int K\left(\frac{x-y}{h}\right) d \hat{F}_{\mathrm{KM}}(y) \\
& =\frac{1}{h} \sum_{j=1}^{n} K\left(\frac{x-W_{j}}{h}\right) s_{j}
\end{aligned}
$$

where $s_{j}$ is the size of jump of $\hat{F}_{\mathrm{KM}}$ at $W_{j}, h=h_{n}$ is a positive bandwidth or window width, s.t. $h \rightarrow 0$ as $n \rightarrow \infty$ and kernel $K$ is a symmetric function satisfying

$$
\int K(t) \mathrm{d} t=1, \quad \int t K(t) \mathrm{d} t=0, \quad \text { and } \int t^{2} K(t) \mathrm{d} t<\infty
$$

Thus from (4) and (6), the K-M type deconvolution kernel density estimator of the unknown density $f_{Z}$ can be written as

$$
\hat{f}_{Z}^{\mathrm{KM}}(z)=\frac{1}{h} \sum_{j=1}^{n} K^{\mathrm{KM}}\left(\frac{z-W_{j}}{h}\right) s_{j}
$$

where

$$
K^{\mathrm{KM}}\left(\frac{z-W_{j}}{h}\right)=\frac{1}{2 \pi} \int \mathrm{e}^{-i y \frac{z-W_{j}}{h}} \frac{\varphi_{K}(y)}{\varphi_{f_{E}\left(\frac{y}{h}\right)}} \mathrm{d} y
$$


The K-M type estimator was used in evaluating the duration of breastfeeding data by Chakrabarty [1]

In this paper, instead of using Kaplan-Meier estimator, we adopt the idea of inverseprobability-of-censoring weighted (IPCW) average by Robins and Finkelstein [12]. As pointed out by Satten and Datta [13], the Kaplan-Meier equivalent estimator $\hat{F}_{\mathrm{IP}}(\cdot)$ can be written as

$$
\hat{F}_{\mathrm{IP}}(x)=\frac{1}{n} \sum_{i=1}^{n} \frac{I\left(W_{i} \leq x\right) \delta_{i}}{1-G\left(W_{i}\right)} .
$$

Therefore, the kernel type estimator for $p d f f_{X}(\cdot)$ can be formulated as

$$
\begin{aligned}
\hat{f}_{X}^{\mathrm{IP}}(x) & =\frac{1}{h} \int K\left(\frac{x-y}{h}\right) \mathrm{d} \hat{F}_{\mathrm{IP}}(y) \\
& =\frac{1}{n h} \sum_{j=1}^{n} K\left(\frac{x-W_{j}}{h}\right) \frac{\delta_{j}}{1-G\left(W_{j}\right)}
\end{aligned}
$$

Consequently, we obtain the new class of deconvolution kernel density estimator of $f_{Z}$ as

$$
\begin{aligned}
\hat{f}_{Z}^{\mathrm{IP}}(z) & =\frac{1}{2 \pi} \int \mathrm{e}^{-\mathrm{i} t z} \frac{\varphi_{f_{X}^{\mathrm{IP}}}(t)}{\varphi_{f_{E}}(t)} \mathrm{d} t \\
& =\frac{1}{n h} \sum_{j=1}^{n} K^{E}\left(\frac{z-W_{j}}{h}\right) \frac{\delta_{j}}{1-G\left(W_{j}\right)},
\end{aligned}
$$

where

$$
K^{E}\left(\frac{z-W_{j}}{h}\right)=\frac{1}{2 \pi} \int \mathrm{e}^{-\mathrm{i} y \frac{z-W_{j}}{h}} \frac{\varphi_{K}(y)}{\varphi_{f_{E}}\left(\frac{y}{h}\right)} \mathrm{d} y
$$

In model (1), when the observations $\left\{X_{j}, j=1,2, \ldots, n\right\}$ are complete (noncensored), estimator of $f_{Z}(\cdot)$ becomes

$$
\hat{f}_{Z}(z)=\frac{1}{n h} \sum_{j=1}^{n} K^{E}\left(\frac{z-X_{j}}{h}\right)
$$

where

$$
K^{E}\left(\frac{z-X_{j}}{h}\right)=\frac{1}{2 \pi} \int \mathrm{e}^{-\mathrm{i} y \frac{z-X_{j}}{h}} \frac{\varphi_{K}(y)}{\varphi_{f_{E}}\left(\frac{y}{h}\right)} \mathrm{d} y
$$

Fan [4] investigated the limiting distribution of the $k$ th derivative of $\hat{f}_{Z}$ given by (13). In another work by Fan and Truong [7], the estimator in (13) was used to estimate 
the regression function. That is, for pair of random variable $(Z, Y)$, considering the problem of estimating the regression function $m(z)=E(Y \mid Z=z)$, where due to the measuring mechanism or the nature of the environment, the variable $Z$ is measured with error $E$ and is not directly observable (Fuller [8]). Instead, $Z$ is contaminated in $X=$ $Z+E, E$ is a random disturbance. Given the random sample $\left\{Y_{j}, j=1,2, \ldots, n\right\}$ and $\left\{X_{j}, j=1,2, \ldots, n\right\}$, Fan and Truong [7] proposed the non-parametric regression estimator as

$$
\hat{m}(z)=\sum_{j} \frac{K^{E}\left(\frac{z-X_{j}}{h}\right) Y_{j}}{\hat{f}_{Z}(z)}
$$

where $\hat{f}_{Z}(\cdot)$ and $K^{E}(\cdot)$ are given by (13) and (14).

\section{Main Theorems}

In this section, we focus on the limiting distribution of $\hat{f}_{Z}^{\mathrm{IP}}(z)$. Following Fan [4], we assume that $\varphi_{f_{E}}$ satisfies one of the following conditions.

Assumption 3.1 (i) Ordinary smooth case:

$$
\varphi_{f_{E}}(t) t^{\beta} \rightarrow c \quad \text { as } t \rightarrow \infty
$$

with some constant $c \neq 0$ and $\beta \geq 0$. Moreover we assume $\varphi_{f_{E}}(t) \neq 0$, for all $t$. Or

(ii) Supersmooth case:

$$
c_{1}|t|^{\beta_{0}} \exp \left(-|t|^{\beta} / \gamma\right) \leq\left|\varphi_{f_{E}}(t)\right| \leq c_{2}|t|^{\beta_{0}} \exp \left(-|t|^{\beta} / \gamma\right) \text { as } t \rightarrow \infty
$$

with $\beta, \gamma, c_{1}, c_{2}>0$ and some real number $\beta_{0} . \varphi_{f_{E}}(t) \neq 0$ for all $t$.

Let $\varphi_{f_{E}}(t)=R_{E}(t)+\mathrm{i} I_{E}(t)$, where $R_{E}(t)$ and $I_{E}(t)$ denote the real and the imaginary part of the characteristic function $\varphi_{f_{E}}(t)$. Assume furthermore that either $I_{E}(t)=o\left(R_{E}(t)\right)$ or $R_{E}(t)=o\left(I_{E}(t)\right)$ as $t \rightarrow \infty$.

\subsection{Case I: Ordinary Smooth Distribution}

We make the following assumptions on the characteristic function $\varphi_{K}$ of kernel function $K$.

Assumption 3.2 (i) $\varphi_{K}(t)$ is a symmetric function, having $s+2$ bounded integrable derivatives, $\varphi_{K}(0)=1$.

(ii) $\int_{-\infty}^{\infty}\left[\left|\varphi_{K}(t)\right|+\left|\varphi_{K}^{\prime}(t)\right|\right]|t|^{\beta} d t<\infty, \int_{-\infty}^{\infty}|t|^{2 \beta}\left|\varphi_{K}(t)\right|^{2} d t<\infty$.

The following theorem gives the asymptotic normality of the estimator $\hat{f}_{Z}^{\mathrm{IP}}(z)$. 
Theorem 3.1 Use Assumption 3.2, if $h=h_{n} \rightarrow 0$ as $n \rightarrow \infty$ and $n h \rightarrow \infty$, then

$$
\frac{\hat{f}_{Z}^{\mathrm{IP}}(z)-E \hat{f}_{Z}^{\mathrm{IP}}(z)}{\sqrt{\operatorname{Var}\left(\hat{f}_{Z}^{\mathrm{IP}}(z)\right)}} \stackrel{d}{\rightarrow} N(0,1) .
$$

The proof of Theorem 3.1 relies on the following lemma, which is a generalization of Theorem 1A of Parzen [10].

Lemma 3.1 Suppose that $K_{n}(\cdot)$ is a sequence of Borel functions satisfying

$$
K_{n}(x) \rightarrow \widetilde{K}(x) \text { as } n \rightarrow \infty \text { and } \sup _{n}\left|K_{n}(x)\right| \leq K^{*}(x)
$$

where $K^{*}(x)$ satisfies

$$
\begin{gathered}
\int_{-\infty}^{\infty} K^{*}(x) \mathrm{d} x<\infty \\
\lim _{x \rightarrow \infty}\left|x K^{*}(x)\right|=0
\end{gathered}
$$

Let $m(x)$ satisfy $\int_{-\infty}^{\infty}|m(x)| \mathrm{d} x<\infty$, if $z$ is a continuity point of $m(\cdot)$, then for any sequence $h=h_{n} \rightarrow 0$ as $n \rightarrow \infty$,

$$
\lim _{n \rightarrow \infty} \frac{1}{h} \int_{-\infty}^{\infty} K_{n}\left(\frac{z-x}{h}\right) m(x) \mathrm{d} x=m(z) \int_{-\infty}^{\infty} \widetilde{K}(x) \mathrm{d} x
$$

Proof of Lemma 3.1 Let $b>0$, and split the region of integration into two parts: $|x| \leq b$ and $|x|>b$, we have

$$
\begin{aligned}
& \left|\frac{1}{h} \int_{-\infty}^{\infty} K_{n}\left(\frac{z-x}{h}\right) m(x) \mathrm{d} x-m(z) \int_{-\infty}^{\infty} \widetilde{K}(x) \mathrm{d} x\right| \\
& \leq\left|\frac{1}{h} \int_{-\infty}^{\infty} K_{n}\left(\frac{x}{h}\right)[m(z-x)-m(z)] \mathrm{d} x\right|+|m(z)|\left|\int_{-\infty}^{\infty}\left(\frac{1}{h} K_{n}\left(\frac{x}{h}\right)-\widetilde{K}(x)\right) \mathrm{d} x\right| \\
& \leq \max _{|x| \leq b}|m(z-x)-m(z)| \int_{-\infty}^{\infty} K^{*}(x) \mathrm{d} x+\int_{|x| \geq b} \frac{|m(z-x)|}{x} \frac{x}{h} K_{n}\left(\frac{x}{h}\right) \mathrm{d} x \\
& \quad+|m(z)| \int_{|x| \geq b} \frac{1}{h} K_{n}\left(\frac{x}{h}\right) \mathrm{d} x+|m(z)|\left|\int_{-\infty}^{\infty}\left(K_{n}(x)-\widetilde{K}(x)\right) \mathrm{d} x\right| \\
& \leq \max _{|x| \leq b}|m(z-x)-m(z)| \int_{-\infty}^{\infty} K^{*}(x) \mathrm{d} x+\frac{1}{b} \sup _{|y| \geq \frac{b}{h}}\left|y K^{*}(y)\right| \int_{-\infty}^{\infty}|m(x)| \mathrm{d} x \\
& \quad+|m(z)| \int_{|y| \geq \frac{b}{h}} K^{*}(y) \mathrm{d} y+|m(z)|\left|\int_{-\infty}^{\infty}\left(K_{n}(x)-\widetilde{K}(x)\right) \mathrm{d} x\right|
\end{aligned}
$$


When $n \rightarrow \infty$, the last three terms of (20) tend to 0 by the assumptions and Lebesgue's dominated convergence theorem. Let $b \rightarrow 0$, the first term also tends to 0 .

We now are ready to prove Theorem 3.1.

Proof of Theorem 3.1 We first rewrite $\hat{f}_{Z}^{\mathrm{IP}}(z)$ in (11) as follows:

$$
\begin{aligned}
& \hat{f}_{Z}^{\mathrm{IP}}(z)=\frac{1}{n h} \sum_{j=1}^{n} K^{E}\left(\frac{z-W_{j}}{h}\right) \frac{\delta_{j}}{1-G\left(W_{j}\right)}=\frac{1}{n} \sum_{j=1}^{n} U_{j}, \\
& \text { where } U_{j}=\frac{\delta_{j}}{h} \frac{K^{E}\left(\frac{z-W_{j}}{h}\right)}{1-G\left(W_{j}\right)} . \\
& \frac{\hat{f}_{Z}^{\mathrm{IP}}(z)-E \hat{f}_{Z}^{\mathrm{IP}}(z)}{\sqrt{\operatorname{Var}\left(\hat{f}_{Z}^{\mathrm{IP}}(z)\right)}}=\frac{\sum_{j=1}^{n}\left(U_{j}-E U_{j}\right)}{\sum_{j=1}^{n} \operatorname{Var}\left(U_{j}\right)} \stackrel{\mathrm{d}}{\rightarrow} N(0,1)
\end{aligned}
$$

Note that (22) holds

if Lyapunov's condition satisfies, i.e., for some $v>0$

$$
\frac{\sum_{j=1}^{n} E\left|U_{j}-E U_{j}\right|^{2+v}}{\left[\sum_{j=1}^{n} \operatorname{Var}\left(U_{j}\right)\right]^{1+v / 2}} \rightarrow 0
$$

By $c_{r}$-inequality Dufour [3], an upper bound of the left side of (23) is

$$
\frac{\sum_{j=1}^{n} E\left|U_{j}-E U_{j}\right|^{2+v}}{\left[\sum_{j=1}^{n} \operatorname{Var}\left(U_{j}\right)\right]^{1+v / 2}} \leq 2^{1+v} \frac{\sum_{j=1}^{n}\left(E\left|U_{j}\right|^{2+v}+\left|E U_{j}\right|^{2+v}\right)}{\left[\sum_{j=1}^{n} \operatorname{Var}\left(U_{j}\right)\right]^{1+v / 2}}
$$

First, we evaluate the limit of $E U_{j}{ }^{2}$. Note that when $\delta=1, P\left(C_{j} \leq W_{j}\right)=$ $G\left(W_{j}\right)=0$, hence

$$
\begin{aligned}
E U_{j}^{2} & =E\left[\frac{1}{h} K^{E}\left(\frac{z-W_{j}}{h}\right) \frac{\delta_{j}}{1-G\left(W_{j}\right)}\right]^{2} \\
& =\frac{1}{h^{2}} \int_{-\infty}^{\infty}\left[K^{E}\left(\frac{z-w}{h}\right)\right]^{2} f_{X \mid \delta=1}(w) p \mathrm{~d} w \\
& =\frac{p}{h^{2+2 \beta}} \int_{-\infty}^{\infty}\left[h^{\beta} K^{E}\left(\frac{z-w}{h}\right)\right]^{2} f_{X \mid \delta=1}(w) \mathrm{d} w
\end{aligned}
$$

where $f_{X \mid \delta=1}$ denotes the conditional density of $X$ given $\delta=1$.

By Assumption 3.1 (i) and Lebesgue's dominated convergence theorem,

$$
h^{\beta} K^{E}(w) \rightarrow \frac{1}{2 \pi c} \int_{-\infty}^{\infty} \mathrm{e}^{-\mathrm{i} y w} y^{\beta} \varphi_{K}(y) \mathrm{d} y, \text { as } n \rightarrow \infty
$$


Applying Plancherel's theorem ([11]) and by Assumption 3.2 (ii), we see that (18) in Lemma 3.1 holds due to following results:

$$
\begin{aligned}
\int_{-\infty}^{\infty}\left[h^{\beta} K^{E}(w)\right]^{2} \mathrm{~d} w & \rightarrow \frac{1}{(2 \pi c)^{2}} \int_{-\infty}^{\infty}\left[\int_{-\infty}^{\infty} \mathrm{e}^{-\mathrm{i} y w} y^{\beta} \varphi_{K}(y) \mathrm{d} y\right]^{2} \mathrm{~d} w \\
& =\frac{1}{2 \pi c^{2}} \int_{-\infty}^{\infty}\left|y^{\beta} \varphi_{K}(y)\right|^{2} \mathrm{~d} y<\infty, \text { as } n \rightarrow \infty
\end{aligned}
$$

Next, (19) in Lemma 3.1 is true due to the fact $\left[h^{\beta} K^{E}(w)\right]^{2} \leq C_{1} / w^{2}$ for some constant $C_{1}$ (Fan [4]). Therefore $\lim _{w \rightarrow \infty}\left|w \cdot\left(C_{1} / w^{2}\right)\right|=0$.

Assuming 3.2 and applying Lemma 3.1 in (25), we obtain

$$
\lim _{n \rightarrow \infty} h^{1+2 \beta} E U_{j}^{2}=p f_{X \mid \delta=1}(z) \int_{-\infty}^{\infty}\left[h^{\beta} K^{E}(w)\right]^{2} \mathrm{~d} w .
$$

Next,

$$
\begin{aligned}
\left|E U_{j}\right| & =\frac{p}{h}\left|\int_{-\infty}^{\infty} K^{E}\left(\frac{z-w}{h}\right) f_{X \mid \delta=1}(w) \mathrm{d} w\right| \\
& =\frac{p}{h}\left|E\left[K^{E}\left(\frac{z-X_{j}}{h}\right) \mid \delta_{j}=1\right]\right|
\end{aligned}
$$

From Stefanski and Carroll [14], Fan [4] and Lemma 3.1 we have,

$$
\begin{aligned}
& \frac{1}{h} E\left[K^{E}\left(\frac{z-X_{j}}{h}\right) \mid \delta_{j}=1\right] \\
& =\frac{1}{h} E\left\{E\left[K^{E}\left(\frac{z-X_{j}}{h}\right) \mid Z_{j}, \delta_{j}=1\right] \mid \delta_{j}=1\right\} \\
& =\frac{1}{h} E\left\{E\left[\frac{1}{2 \pi} \int_{-\infty}^{\infty} \mathrm{e}^{-\mathrm{i} y \frac{z-X_{j}}{h}} \frac{\varphi_{K}(y)}{\varphi_{f_{E}}\left(\frac{y}{h}\right)} \mathrm{d} y \mid Z_{j}, \delta_{j}=1\right] \mid \delta_{j}=1\right\} \\
& =\frac{1}{2 \pi h} E\left\{E\left[\int_{-\infty}^{\infty} \mathrm{e}^{-\mathrm{i} y \frac{z-\left(Z_{j}+E_{j}\right)}{h}} \frac{\varphi_{K}(y)}{\varphi_{f_{E}}\left(\frac{y}{h}\right)} \mathrm{d} y \mid Z_{j}, \delta_{j}=1\right] \mid \delta_{j}=1\right\} \\
& =\frac{1}{2 \pi h} \int_{-\infty}^{\infty} \mathrm{e}^{\mathrm{i} y \frac{E_{j}}{h}} \frac{\varphi_{K}(y)}{\varphi_{f_{E}}\left(\frac{y}{h}\right)} E\left\{E\left[\mathrm{e}^{-\mathrm{i} y \frac{z-Z_{j}}{h}} \mid Z_{j}, \delta_{j}=1\right] \mid \delta_{j}=1\right\} \mathrm{d} y \\
& =\frac{1}{2 \pi h} \int \varphi_{K}(y) E\left[\mathrm{e}^{-\mathrm{i} y \frac{z-z_{j}}{h}} \mid \delta_{j}=1\right] \mathrm{d} y \\
& =\frac{1}{2 \pi h} E\left[\int_{-\infty}^{\infty} \varphi_{K}(y) \mathrm{e}^{-i y \frac{z-Z_{j}}{h}} \mathrm{~d} y \mid \delta_{j}=1\right] \\
& =\frac{1}{h} E\left[K\left(\frac{z-Z_{j}}{h}\right) \mid \delta_{j}=1\right]=\frac{1}{2 \pi h} \int_{-\infty}^{\infty} K\left(\frac{z-x}{h}\right) f_{Z \mid \delta=1}(x) \mathrm{d} x \\
& \rightarrow f_{Z \mid \delta=1}(z) \int_{-\infty}^{\infty} K(x) \mathrm{d} x=f_{Z \mid \delta=1}(z), \text { as } n \rightarrow \infty
\end{aligned}
$$


where $f_{Z \mid \delta=1}$ denotes the conditional density of $Z$ given $\delta=1$.

Thus

$$
\left|E U_{j}\right|=\mathcal{O}(1)
$$

Similarly,

$$
E\left|U_{j}\right|^{2+v}=\frac{p}{h^{(2+v)(1+\beta)}} \int_{-\infty}^{\infty}\left[h^{\beta} K^{E}\left(\frac{z-w}{h}\right)\right]^{2+v} f_{X \mid \delta=1}(w) \mathrm{d} w
$$

Note that $\int_{\mathbb{R}}\left|h^{\beta} K^{E}(u)\right|^{2+v} \mathrm{~d} u \leq C(1+o(1))$, where $C$ is some positive constant. By Lemma 2 in Masry [9] we have

$$
\lim _{n \rightarrow \infty} h^{(2+v)(1+\beta)-1} E\left|U_{j}\right|^{2+v}=p f_{X \mid \delta=1}(z) \int_{-\infty}^{\infty}\left[h^{\beta} K^{E}(w)\right]^{2+v} \mathrm{~d} w .
$$

Therefore

$$
E\left|U_{j}\right|^{2+v}=\mathcal{O}\left(h^{-(2+v)(1+\beta)+1}\right) .
$$

Consequently, Lyapunov's condition (23) follows by (24), (30) and (32), we have

$$
\frac{\sum_{j=1}^{n} E\left|U_{j}-E U_{j}\right|^{2+v}}{\left[\sum_{j=1}^{n} \operatorname{Var}\left(U_{j}\right)\right]^{1+v / 2}}=\mathcal{O}\left(1 /(n h)^{v / 2}\right) \rightarrow 0, \text { as } n \rightarrow \infty .
$$

Remark The asymptotic normality for $\hat{f}_{Z}^{\mathrm{IP}}(z)$ in Theorem 3.1 contains the unknown $\operatorname{Var}\left(\hat{f}_{Z}^{\mathrm{IP}}(z)\right)$, where

$$
\operatorname{Var}\left(\hat{f}_{Z}^{\mathrm{IP}}(z)\right)=\frac{1}{2 \pi c^{2}} \frac{p}{n h^{2 \beta+1}} f_{X \mid \delta=1}(z) \int_{-\infty}^{\infty}|y|^{2 \beta}\left|\varphi_{K}(y)\right|^{2} \mathrm{~d} y(1+o(1))
$$

One can estimate $\operatorname{Var}\left(\hat{f}_{Z}^{\mathrm{IP}}(z)\right)$ with $S_{n}^{2}=\sum_{j=1}^{n} U_{j}^{2} / n$ or the sample variance $S_{n}^{2}=\sum_{j=1}^{n} \frac{1}{n}\left(U_{j}-\frac{1}{n} \sum_{j=1}^{n} U_{j}\right)^{2}, U_{j}$ is given in (21). Replacing $\operatorname{Var}\left(\hat{f}_{Z}^{\mathrm{IP}}(z)\right)$ in Theorem 3.1 with its estimator $S_{n}^{2}$, we have the following Corollary:

Corollary 3.1 Under Assumption 3.2, if $h=o\left(n^{-1 /(2 s+2 \beta+1)}\right)$, then

$$
\sqrt{n} \frac{\hat{f}_{Z}^{\mathrm{IP}}(z)-p f_{Z \mid \delta=1}(z)}{S_{n}} \stackrel{d}{\rightarrow} N(0,1)
$$

where $S_{n}$ is given by either $S_{n}^{2}=\sum_{j=1}^{n} \frac{1}{n} U_{j}^{2}$ or the sample variance defined by $S_{n}^{2}=\sum_{j=1}^{n} \frac{1}{n}\left(U_{j}-\frac{1}{n} \sum_{j=1}^{n} U_{j}\right)^{2}$ provided $n h^{2 \beta+1} \rightarrow \infty$. 
To prove Corollary 3.1, we need the following Lemma.

Lemma 3.2 Under the assumption of Theorem 3.1,

$$
\frac{\sum_{j=1}^{n} U_{j}^{2}}{n E U_{1}^{2}} \stackrel{P}{\rightarrow} 1
$$

and if $n h^{2 \beta+1} \rightarrow \infty$ as $n \rightarrow \infty$ then

$$
\hat{f}_{Z}^{\mathrm{IP}}(z)-E \hat{f}_{Z}^{\mathrm{IP}}(z)=\frac{1}{n} \sum_{j=1}^{n} U_{j}-E U_{1} \stackrel{P}{\rightarrow} 0
$$

Proof of Lemma 3.2 By Corollary 2 in Teicher and Chow [15], let $Y_{n j}=U_{j}^{2} /\left(n E U_{j}^{2}\right)$, $\sum_{j=1}^{n} E Y_{n j}=1$ then $\sum_{j=1}^{n} Y_{n j} \stackrel{\mathrm{P}}{\rightarrow} 1$ iff $\sum_{j=1}^{n} E\left(Y_{n j} I_{\left\{Y_{n j} \geq \epsilon\right\}}\right)=o(1), \epsilon>0$. i.e., (36) holds if for each $\epsilon>0$ we have

$$
\frac{\sum_{j=1}^{n} E\left(U_{j}^{2} I_{\left\{U_{j}^{2} /\left(n E U_{j}^{2}\right) \geq \epsilon\right\}}\right)}{n E U_{j}^{2}}=\frac{E\left(\left(U_{j}^{2} I_{\left\{U_{j}^{2} / E U_{j}^{2} \geq n \epsilon\right\}}\right)\right.}{E U_{j}^{2}} \rightarrow 0, \text { as } n \rightarrow \infty
$$

Note that $\left|h^{\beta} K^{E}(w)\right|^{2} \leq C_{2}^{2}$, we have

$$
U_{j}^{2}=\frac{1}{h^{2}}\left[K^{E}\left(\frac{z-W_{j}}{h}\right) \frac{\delta_{j}}{1-G\left(W_{j}\right)}\right]^{2}=\mathcal{O}_{p}\left(C_{2}^{2} / h^{2 \beta+2}\right)
$$

By (27), $E U_{j}^{2}=h^{-2 \beta-1} C_{3}(1+o(1))$, where $C_{3}$ is some positive constant. Thus we have

$U_{j}^{2} /\left(n E U_{j}^{2}\right) \leq \epsilon$ with probability 1. Hence, $I_{\left\{U_{j}^{2} / E U_{j}^{2} \geq n \epsilon\right\}}=0$ as $n \rightarrow \infty$ with probability 1 . Therefore (38) holds.

By (34), if $n h^{2 \beta+1} \rightarrow \infty$, we obtain

$$
n^{-1} \operatorname{Var}\left(U_{j}\right)=\mathcal{O}\left(n^{-1} h^{-2 \beta-1}\right) \rightarrow 0
$$

which implies (37).

\section{Proof of Corollary 3.1}

$$
\begin{aligned}
E \hat{f}_{Z}^{\mathrm{IP}}(z) & =E U_{j}=\frac{1}{h} E\left[K^{E}\left(\frac{z-W_{j}}{h}\right) \frac{\delta_{j}}{1-G\left(W_{j}\right)}\right] \\
& =\frac{p}{h} \int_{-\infty}^{\infty} K^{E}\left(\frac{z-w}{h}\right) f_{X \mid \delta=1}(w) \mathrm{d} w \\
& =\frac{p}{h} E\left[K^{E}\left(\frac{z-Z_{j}}{h}\right) \mid \delta_{j}=1\right]
\end{aligned}
$$


Using (29), we have

$$
\begin{aligned}
& \left|E \hat{f}_{Z}^{\mathrm{IP}}(z)-p f_{Z \mid \delta=1}(z)\right| \\
& \quad \leq p\left|\frac{1}{h} \int_{-\infty}^{\infty} K^{E}\left(\frac{z-w}{h}\right)\left(f_{X \mid \delta=1}(w) \mathrm{d} w-f_{Z \mid \delta=1}(z)\right)\right| \rightarrow 0
\end{aligned}
$$

When $S_{n}^{2}=\frac{1}{n} \sum_{j=1}^{n} U_{j}^{2}$ or $S_{n}^{2}=\frac{1}{n} \sum_{j=1}^{n}\left(U_{j}-\frac{1}{n} \sum_{j=1}^{n} U_{j}\right)^{2}$, by Slutsky's theorem, (35) holds by (36) and (42).

\subsection{Case II: Supersmooth Distribution}

In this subsection we assume that $\varphi_{f_{E}}$ satisfies Assumption 3.1 (ii) and $\varphi_{K}$ satisfies the following assumption.

Assumption $3.3 \varphi_{K}(t)$ is a symmetric function, supported with $[-1,1]$ and having the first $s+2$ continuous derivatives, $\varphi_{K}(t)>c_{3}(1-t)^{s+3}$, for $t \in[1-\psi, 1)$ for some $\psi>0$, and $c_{3}>0$.

Following theorem states the limiting distribution of $\hat{f}_{Z}^{\mathrm{IP}}(z)$ when the error distribution is supersmooth.

Theorem 3.2 Under Assumption 3.3, we have

$$
\frac{\hat{f}_{Z}^{\mathrm{IP}}(z)-E \hat{f}_{Z}^{\mathrm{IP}}(z)}{\sqrt{\operatorname{Var}\left(\hat{f}_{Z}^{\mathrm{IP}}(z)\right)}} \stackrel{d}{\rightarrow} N(0,1) .
$$

provided $h=h_{n}=a(\ln n)^{-1 / \beta}$, for some $a>0$.

Before we proceed with the proof of Theorem 3.2, we need the following lemma from Fan [4].

Lemma 3.3 Under Assumption 3.3, as $n \rightarrow \infty$,

$$
\left|K^{E}(x)\right| \geq c_{4} q(x) \exp \left(\frac{\left(1-b_{n}\right)^{\beta}}{\gamma h^{\beta}}\right) h^{\beta_{0}} b_{n}^{s+4}
$$

uniformly over $x \in[0, \pi / 2]$, where $h=h_{n}, b_{n}=h_{n}^{\beta /(2(s+5))}$ and $c_{4}$ is a positive constant, and

$$
q(x)= \begin{cases}|\cos x|, & \text { if } I_{E}(t)=o\left(R_{E}(t)\right) \\ |\sin x|, & \text { if } R_{E}(t)=o\left(I_{E}(t)\right)\end{cases}
$$

where $R_{E}(t)$ and $I_{E}(t)$ denote the real and the imaginary part of the characteristic function $\varphi_{f_{E}}(t)$. 
Proof of Theorem 3.2 Check that (23) is the sufficient condition of (43).

Note that by (24),

$$
\frac{\sum_{j=1}^{n} E\left|U_{j}-E U_{j}\right|^{2+v}}{\left[\sum_{j=1}^{n} \operatorname{Var}\left(U_{j}\right)\right]^{1+v / 2}} \leq 2^{1+v} \frac{\sum_{j=1}^{n}\left(E\left|U_{j}\right|^{2+v}+\left|E U_{j}\right|^{2+v}\right)}{\left[\sum_{j=1}^{n} E U_{j}^{2}\right]^{1+v / 2}}
$$

By Lemma 3.3, when $n$ is sufficiently large, the lower bound of $E U_{j}^{2}$ can be obtained as follows.

$$
\begin{aligned}
E U_{j}^{2} & =\frac{p}{h} \int_{-\infty}^{\infty}\left[K^{E}(t)\right]^{2} f_{X \mid \delta=1}(z-h t) \mathrm{d} t \\
& \geq \frac{p}{h} \int_{0}^{\frac{\pi}{2}}\left[c_{4} q(x) \exp \left(\frac{\left(1-b_{n}\right)^{\beta}}{\gamma h^{\beta}}\right) h^{\beta_{0}} b_{n}^{s+4}\right]^{2} f_{X \mid \delta=1}(z-h t) \mathrm{d} t \\
& =\frac{p}{h}\left[c_{4} \exp \left(\frac{\left(1-b_{n}\right)^{\beta}}{\gamma h^{\beta}}\right) h^{\beta_{0}} b_{n}^{s+4}\right]^{2} \int_{0}^{\frac{\pi}{2}} q^{2}(t) f_{X \mid \delta=1}(z-h t) \mathrm{d} t \\
& \geq c_{5} h^{c_{6}} f_{X \mid \delta=1}(z) \exp \left(\frac{2\left(1-b_{n}\right)^{\beta}}{\gamma h^{\beta}}\right)
\end{aligned}
$$

Recall that $b_{n}=h_{n}^{\beta /(2(s+5))}$ and $h=a(\ln n)^{-1 / \beta}$, we obtain $b_{n}=a^{\beta /(2 s+10)}(\ln n)^{-1 /(2 s+10)} \rightarrow 0$, as $n \rightarrow \infty$.

According to Taylor's theorem, $\left(1-b_{n}\right)^{\beta}=1-\beta b_{n}+R\left(b_{n}\right)$, where $R\left(b_{n}\right)=o\left(b_{n}\right)$. It follows that $\left(1-b_{n}\right)^{\beta} \approx 1-\beta b_{n} \geq 1-2 \beta b_{n}$. Thus,

$$
E U_{j}^{2} \geq c_{5} f_{X \mid \delta=1}(z) h^{c_{6}} \exp \left(\frac{2-4 \beta b_{n}}{\gamma h^{\beta}}\right)
$$

for some constant $c_{5}>0$ and $c_{6}=2 \beta_{0}-1+\beta(s+4) /(s+5)$.

Next we will find the upper bound of $E U_{j}$ and $E\left|U_{j}\right|^{2+\delta}$ by using the upper bound for $\left|K^{E}\right|$ provided in Fan [4]: $\left|K^{E}(t)\right| \leq \mathcal{O}\left(\exp \left(1 /\left(\gamma h^{\beta}\right)\right) c_{n}\right)$, where $h=h_{n}$,

$$
c_{n}= \begin{cases}1, & \beta_{0} \geq 0 \\ h_{n} \beta_{0}, & \beta_{0}<0\end{cases}
$$

Note that $\left|E U_{j}\right|$ is bounded as shown in (30). Moreover,

$$
\begin{aligned}
E\left|U_{j}\right|^{2+v} & =E\left|\frac{1}{h} K^{E}\left(\frac{z-W_{j}}{h}\right) \frac{\delta_{j}}{1-G\left(W_{j}\right)}\right|^{2+v} \\
& =\frac{p}{h^{2+v}} \int_{-\infty}^{\infty}\left[K^{E}\left(\frac{z-w}{h}\right)\right]^{2+v} f_{X \mid \delta=1}(w) \mathrm{d} w \\
& =\frac{p}{h^{2+v}} \int_{-\infty}^{\infty}\left[K^{E}\left(\frac{z-w}{h}\right)\right]^{2+v} f_{X \mid \delta=1}(w) \mathrm{d} w
\end{aligned}
$$




$$
\begin{aligned}
& \leq \frac{p}{h^{2+v}} \mathcal{O}\left(\exp \left(\frac{2+v}{\gamma h^{\beta}}\right) c_{n}{ }^{2+v}\right) \int_{-\infty}^{\infty} f_{X \mid \delta=1}(w) \mathrm{d} w \\
& =\mathcal{O}\left(h^{-(2+v)} \exp \left(\frac{2+v}{\gamma h^{\beta}}\right) c_{n}^{2+v}\right) .
\end{aligned}
$$

Hence, by (45),

$$
\frac{\sum_{j=1}^{n} E\left|U_{j}-E U_{j}\right|^{2+v}}{\left[\sum_{j=1}^{n} \operatorname{Var}\left(U_{j}\right)\right]^{1+v / 2}} \rightarrow 0, \text { as } n \rightarrow \infty
$$

by choosing $h=a(\ln n)^{-1 / \beta}$, and for some $a$

such that $a>(2 / \gamma)^{1 / \beta}$.

Similar to ordinary smooth error case, we can use $S_{n}^{2}=\sum_{j=1}^{n} U_{j}^{2} / n$ or $S_{n}^{2}=$ $\frac{1}{n} \sum_{j=1}^{n}\left(U_{j}-E U_{j}\right)^{2}$ to estimate $n \operatorname{Var}\left(\hat{f}_{Z}^{\mathrm{IP}}(z)\right)$. The proofs of Lemma 3.4 and Corollary 3.2 are similar to Lemma 3.2 and Corollary 3.1.

Lemma 3.4 Under Assumption 3.3, if $h=h_{n}=a(\ln n)^{-1 / \beta}$ for some $a>0$ then

$$
\frac{\sum_{j=1}^{n} U_{j}^{2}}{n E U_{j}^{2}} \stackrel{P}{\rightarrow} 1 \text {, as } n \rightarrow \infty
$$

and if $h=((c \gamma \ln n) / 2)^{-1 / \beta}$ for some $0<c<1$ then

$$
\frac{1}{n} \sum_{j=1}^{n} U_{j}-E U_{j} \stackrel{P}{\rightarrow} 0 \text {, as } n \rightarrow \infty
$$

Corollary 3.2 Under Assumption 3.3, if $h=((c \gamma \ln n) / 2)^{-1 / \beta}$ for some $c>1$, then

$$
\sqrt{n} \frac{\hat{f}_{Z}^{\mathrm{IP}}(z)-p f_{Z \mid \delta=1}(z)}{S_{n}} \stackrel{d}{\rightarrow} N(0,1)
$$

where $S_{n}^{2}=\sum_{j=1}^{n} U_{j}^{2} / n$.

\section{Simulation Results}

In this section, we first construct the proposed estimators by simulating data from two target densities, that is Weibull distribution or Gamma distribution.

\section{Target Distribution 1: Weibull Distribution}

$$
\begin{array}{r}
Z \sim \text { Weibull }(\text { shape }=5, \text { scale }=1) \\
C \sim \text { Weibull }(\text { shape }=2.1, \text { scale }=1.5)
\end{array}
$$




\section{Target Distribution 2: Gamma Distribution}

$$
\begin{aligned}
& Z \sim \operatorname{Gamma}(\text { shape }=6, \text { scale }=0.1) \\
& C \sim \operatorname{Gamma}(\text { shape }=1, \text { scale }=0.8)
\end{aligned}
$$

To study the effect of the error distribution on the estimator $\hat{f}_{Z}^{\mathrm{IP}}(z)$, random variable $E$ is taken to be Normal (super smooth error distribution) or Double exponential (ordinary smooth error distribution) as follows:

Example 1 Error $E$ follows $N(0,0.05)$ :

The reason to choose 0.05 as the variance of the normal error distribution is discussed in Fan [6], where Fan argued that when the variance of the error distribution is sufficiently small, one can choose kernel $K$ as a Gaussian kernel. Hence the corresponding deconvolution kernel function $K^{Z}(x)$ has the following form:

$$
K^{Z}(x)=\frac{1}{\sqrt{2 \pi\left(1-\frac{\sigma_{0}^{2}}{h^{2}}\right)}} \exp \left(-\frac{x^{2}}{2\left(1-\frac{\sigma_{0}^{2}}{h^{2}}\right)}\right)
$$

where variance of error distribution $\sigma_{0}^{2}$ in (54) and (59) is chosen so that the reliability ratio (Fuller [8])

$$
r=\frac{\operatorname{Var}(Z)}{\sigma_{0}^{2}+\operatorname{Var}(Z)}=\frac{\operatorname{Var}(X)-\sigma_{0}^{2}}{\operatorname{Var}(X)}=\frac{\sigma_{X}^{2}-\sigma_{0}^{2}}{\sigma_{X}^{2}} \approx \frac{s_{X}^{2}-\sigma_{0}^{2}}{s_{X}^{2}}=0.7
$$

Example 2 Error $E$ follows Truncated Double Exponential distribution:

Since $E$ follows a truncated double exponential distribution, with $f_{E}$ as

$$
f_{E}(u)=\frac{1}{\sigma_{E}} \mathrm{e}^{\frac{-u}{\sigma_{E}}},
$$

where $u$ is non-negative, thus characteristic function of $E$ is given by

$$
\begin{aligned}
\varphi_{f_{E}}(t) & =\int_{0}^{\infty} \mathrm{e}^{i t u} f_{E}(u) \mathrm{d} u \\
& =\frac{1}{1-i \sigma_{E} t}
\end{aligned}
$$

Hence, deconvoluted kernel estimator can be written as

$$
\begin{aligned}
K^{Z}(x) & =\frac{1}{2 \pi} \int_{-\infty}^{\infty} \mathrm{e}^{-i y x} \frac{\varphi_{K}(y)}{\varphi_{f_{E}}\left(\frac{y}{h}\right)} \mathrm{d} y \\
& =\frac{1}{2 \pi} \int_{-\infty}^{\infty} \mathrm{e}^{-i y x} \varphi_{K}(y)\left(1-i \sigma_{E} \frac{y}{h}\right) \mathrm{d} y \\
& =K(x)+\frac{\sigma_{E}}{h} K^{\prime}(x)
\end{aligned}
$$


Table 1 MISE for Weibull distribution

\begin{tabular}{lllllll}
\hline & Normal error & & \multicolumn{3}{c}{ Double exponential error } \\
\hline$K^{Z}(x)$ & $n=200$ & $n=400$ & $n=500$ & $n=200$ & $n=400$ & $n=500$ \\
\hline In (54) & 0.0343 & 0.0257 & 0.0198 & 0.0301 & 0.0214 & 0.0215 \\
In (59) & 0.0269 & 0.0191 & 0.0195 & 0.0245 & 0.0231 & 0.0182 \\
\hline
\end{tabular}

With Kernel $K$ chosen to be Gaussian, the deconvoluted kernel can be written explicitly as:

$$
K^{Z}(x)=\frac{1}{\sqrt{2 \pi}} \exp \left(-\frac{x^{2}}{2}\right)\left[1-\frac{\sigma_{0}^{2}}{2 h^{2}}\left(x^{2}-1\right)\right]
$$

where $\sigma_{0}^{2}$ is given by (55).

Note that, in (11) the censoring distribution $G(\cdot)$ is unknown, we use the KaplanMeier estimator to estimate $G(\cdot)$ (Wang et al. [16]).

$$
\hat{G}(t)=1-\prod_{j: W_{j}<t}\left(\frac{n-R_{j}}{n-R_{j}+1}\right)^{\left(1-\delta_{j}\right)}
$$

where $R_{j}$ is the rank of $W_{j}$ for $j=1,2, \ldots, n$.

It is well known that the choice of the kernel function $K$ has little impact on the estimates whereas, the choice of bandwidth $h$ affects the estimates. Our simulation selects the bandwidth between 0.03 and 0.4 with an increment of 0.01 and finds the optimal value of $h$ that minimizes the MISE. The censoring rates for Weibull distribution are approximately $67 \%, 68 \%$ and $69 \%$ for $n=200,400$ and 500, respectively. The censoring rates for Gamma distribution are approximately $41 \%, 47 \%$ and $48 \%$ for $n=200,400$ and 500, respectively.

Figures $1,2,3$, and 4 provide $\hat{f}_{Z}^{\mathrm{IP}}(z)$ for two target $f_{Z}(z)$ (Weibull and Gamma distributions), with $n=200,400$ and 500. The dotted lines represent the true $p d f \mathrm{~s}$ $f_{Z}(z)$ and the solid lines are the simulated estimators $\hat{f}_{Z}^{\mathrm{IP}}(z)$.

Tables 1 and 2 below provide the average mean integrated squared error (MISE) between $\hat{f}_{Z}^{\mathrm{IP}}(z)$ and the target density (Weibull distribution in Table 1 and Gamma distribution in Table 2) for different sample $\operatorname{sizes}(n=200,400$ and 500) with 100 replications.

In Tables 1 and 2, the MISE for deconvolution kernel with double exponential error given by (59) is smaller than the deconvoluted kernel with normal errors given by (54). Furthermore, as $n$ increases, the MISE decreases faster for double exponential errors than normal errors. These results are similar to the results obtained for other deconvolution estimators, such as the deconvolution kernel regression model discussed by Fan and Truong [7] and theoretical results shown by Fan [5] regarding convergence rates of non-parametric deconvolution estimators when censoring was not considered. 

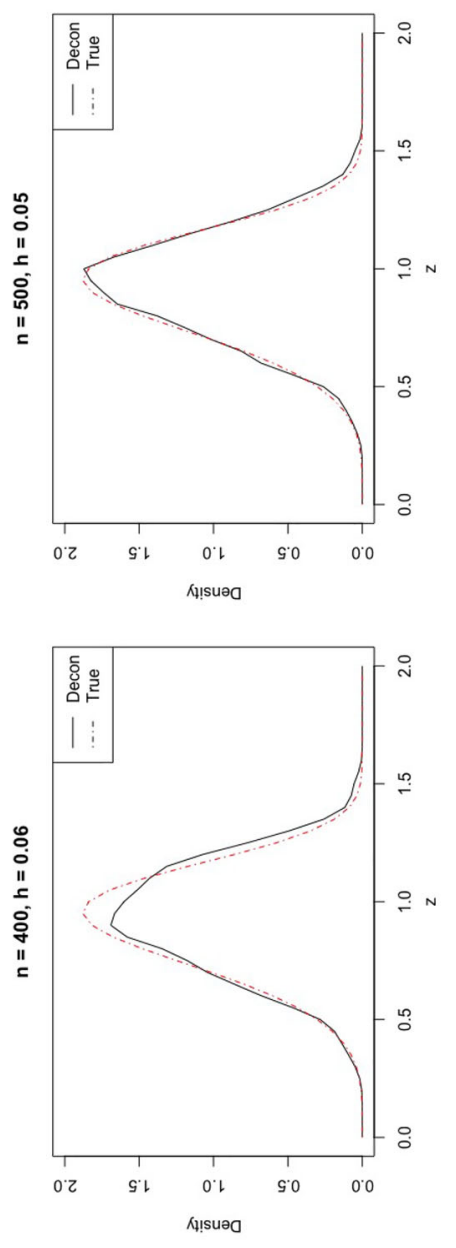

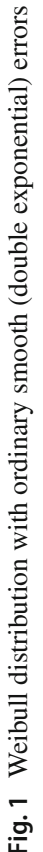



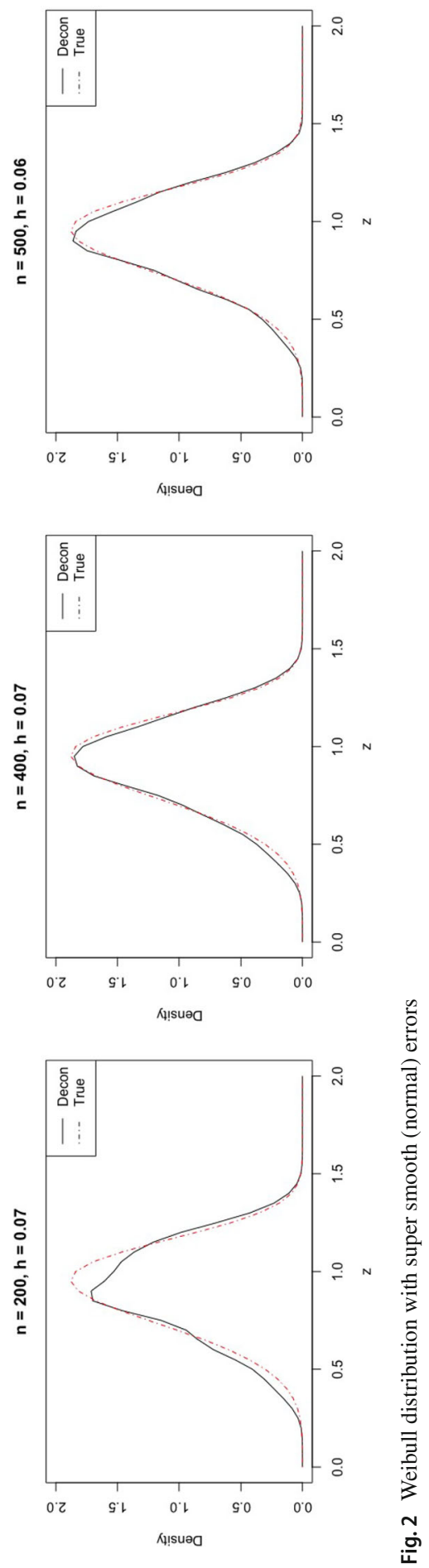

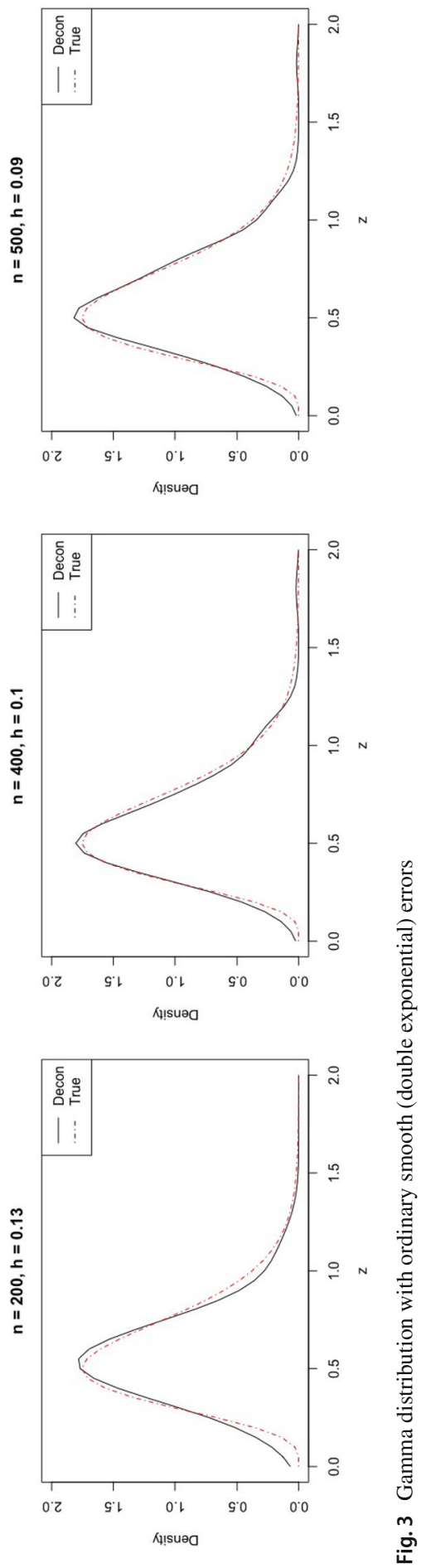

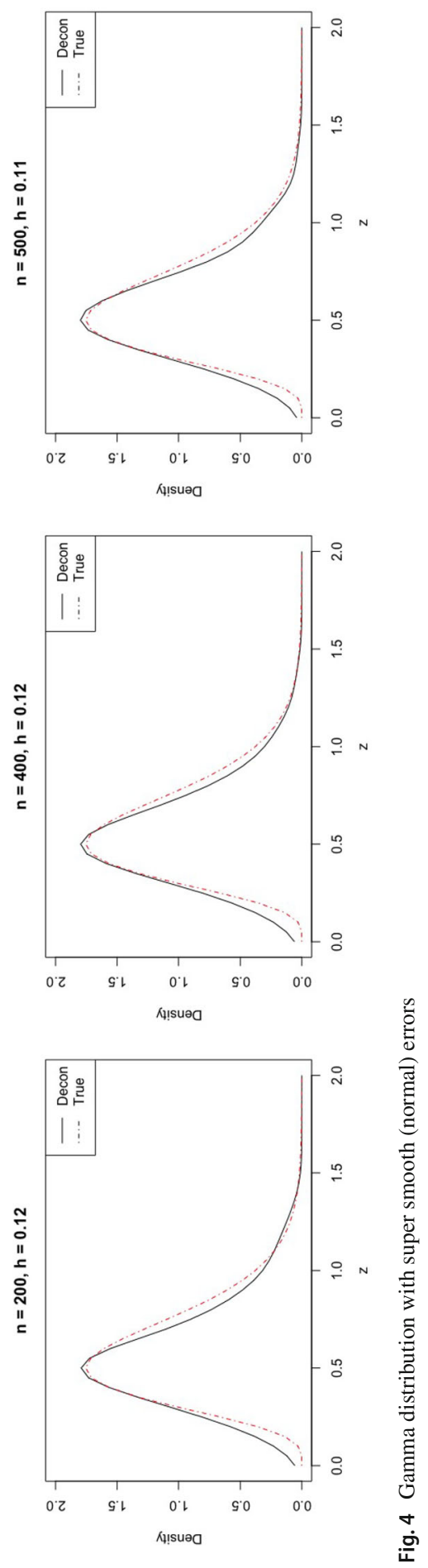
Table 2 MISE for Gamma distribution

\begin{tabular}{lllllll}
\hline & Normal error & & \multicolumn{3}{c}{ Double exponential error } \\
\hline$K^{Z}(x)$ & $n=200$ & $n=400$ & $n=500$ & $n=200$ & $n=400$ & $n=500$ \\
\hline In (54) & 0.0260 & 0.0189 & 0.0187 & 0.0311 & 0.0246 & 0.0147 \\
In (59) & 0.0246 & 0.0165 & 0.0146 & 0.0241 & 0.0163 & 0.0124 \\
\hline
\end{tabular}

Acknowledgements We thank the referees for their constructive comments and suggestions, which improve the manuscript.

\section{References}

1. Chakrabarty, T.: Deconvolving kernel density estimation of right censored duration data with recall errors. Am. J. Appl. Math. Stat. 2(6), 416-422 (2014)

2. Comte, F., Samson, A., Stirnemann, J.J.: Hazard estimation with censoring and measurement error: application to length of pregnancy. TEST 27(2), 338-359 (2018)

3. Dufour, J.-M.: Properties of moments of random variables. Cours de l'Université de Montréal (2003)

4. Fan, J.: Asymptotic normality for deconvolution kernel density estimators. Sankhyā: Indian J. Stat. Ser. A 59, 97-110 (1991)

5. Fan, J.: On the optimal rates of convergence for nonparametric deconvolution problems. Ann. Stat. 19, 1257-1272 (1991)

6. Fan, J.: Deconvolution with supersmooth distributions. La Revue Canadienne de Statistique [Canad. J. Stat.] 20(2), 155-169 (1992)

7. Fan, J., Truong, Y.K.: Nonparametric regression with errors in variables. Ann. Stat. 21, 1900-1925 (1993)

8. Fuller, W.A.: Measurement Error Models, vol. 305. Wiley, New York (2009)

9. Masry, E.: Multivariate probability density deconvolution for stationary random processes. IEEE Trans. Inf. Theory 37(4), 1105-1115 (1991)

10. Parzen, E.: On estimation of a probability density function and mode. Ann. Math. Stat. 33(3), 10651076 (1962)

11. Plancherel, M., Leffler, M.: Contribution à l'étude de la représentation d'une fonction arbitraire par des intégrales définies. Rendiconti del Circolo Matematico di Palermo (1884-1940) 30(1), 289-335 (1910)

12. Robins, J.M., Finkelstein, D.M.: Correcting for noncompliance and dependent censoring in an aids clinical trial with inverse probability of censoring weighted (ipcw) log-rank tests. Biometrics 56(3), 779-788 (2000)

13. Satten, G.A., Datta, S.: The Kaplan-Meier estimator as an inverse-probability-of-censoring weighted average. Am. Stat. 55(3), 207-210 (2001)

14. Stefanski, L.A., Carroll, R.J.: Deconvolving kernel density estimators. Statistics 21(2), 169-184 (1990)

15. Teicher, H., Chow, Y.S.: Probability Theory: Independence, Interchangeability, Martingales. Springer, New York (1978)

16. Wang, Q., Liu, W., Liu, C.: Probability density estimation for survival data with censoring indicators missing at random. J. Multivar. Anal. 100(5), 835-850 (2009)

Publisher's Note Springer Nature remains neutral with regard to jurisdictional claims in published maps and institutional affiliations. 